Original article

\title{
Hypertension and its determinants among school going adolescents in selected urban slums of Nagpur city, Maharashtra: A cross-sectional study
}

\author{
Sitikantha Banerjee $^{\text {a, }}$ Mubashshera F. Khan ${ }^{\text {a }}$, Kajari Bandyopadhyay ${ }^{\text {b, }}$, Kalaiselvi Selvaraj ${ }^{\text {a }}$, \\ Pradeep Deshmukh ${ }^{\text {a }}$
}

${ }^{a}$ Department of Community Medicine, All India Institute of Medical Sciences Nagpur, Plot No. 2, Sector - 20, MIHAN, Nagpur, Maharashtra, 441108, India

${ }^{\mathrm{b}}$ Department of Community Medicine, College of Medicine \& Sagore Dutta Hospital, 578, B.T. Road, Kamarhati, Kolkata, 700058, India

\section{A R T I C L E I N F O}

\section{Keywords:}

Adolescent

Hypertension

Slum

\begin{abstract}
A B S T R A C T
Problem considered: Early detection of hypertension and its risk factors are considered the best possible intervention to avoid complications later in life. Our study was conducted to assess the prevalence of hypertension and its determinants among adolescents of selected schools in slums of Nagpur city, India.

Methods: A cross-sectional study was carried out among 910 school students of Class-VI to IX studying at selected government schools of Nandanvan area, Nagpur city, Central India. A questionnaire for diet, behaviour and mental health was adapted from the Global School Health Survey Questionnaire. Children were considered hypertensive if the systolic or diastolic BP or both were $\geq 95$ th centile considering height, age and sex.

Results: We found $10.6 \%$ and $12.9 \%$ were pre-hypertensive and hypertensive respectively. Inadequate physical activity and a sedentary lifestyle were reported in $62.3 \%$ and $22.7 \%$. In adjusted analysis, father's occupation, family history of Hypertension, mental health symptoms and overweight had significantly increased odds of developing hypertension.

Conclusion: As prevalence of hypertension and its risk factors are high amongst the school students, the need to develop school based focused intervention is paramount.
\end{abstract}

\section{Introduction}

In the last two decades, India is experiencing a significant epidemiological transition, with a constant increase in the burden of noncommunicable diseases (NCDs), ${ }^{1}$ of which, hypertension (HT) contributes a major share. In India, HT is also the most important risk factor for the development of cardio-vascular and cerebro-vascular disease. ${ }^{2}$ Long-standing undiagnosed or untreated HT existing for more than five years may result in target organ damage such as hypertensive retinopathy, stroke and chronic kidney disease. ${ }^{3}$ Its prevalence is increasing in the younger population, where globally essential hypertension in adolescents varies among diverse ethnicities from 0.3 to $21 \%{ }^{4}$ The emerging burden of childhood obesity, ${ }^{5,6}$ early development of atherosclerosis and stress increases the risk of essential HT at a much earlier age group. ${ }^{7,8}$ Based on current evidence, though secondary HT is more common in children (the most common cause being renal disease), primary or essential HT is commoner than secondary HT in adolescents, ${ }^{3}$ which are attributed to multiple risk factors. The asymptomatic nature of HT in the early phases of its onset during adolescence increases the chances of developing complications during adulthood. On the other hand, the roots of essential HT in adults might have initiated in childhood because of its risk factors. Some outcomes associated with hypertension, previously thought confined to adults, are now affecting adolescents also. Studies confirm that the common risk factors associated with hypertension in adults do make similar impact in adolescents too. ${ }^{9}$ Hence hypertension can no longer be considered an adult onset disease. The development of HT and its risk factors are more pertinent in adolescents residing in urban slums, because of their socio-cultural vulnerability i.e. illiteracy, poverty and poor living condition. Thus an earlier detection of HT and identifying its risk factors in adolescence is of potential value. Moreover adolescence is a receptive age group, where behaviour change can be successfully implemented.

However, there is a huge gap in identification of HT among adolescents owing to lack of awareness and competency in diagnosis among health care providers. To the best of our knowledge published literature on HT among this particular age group is not available in the current

\footnotetext{
* Corresponding author.

E-mail address: dr.kajari@gmail.com (K. Bandyopadhyay).
} 
study settings. With this background this study was undertaken to find out the prevalence of hypertension and its determinants among school going adolescents of selected schools of Nagpur city, Central India.

\section{Methods}

\section{Setting and design}

Ours was a cross-sectional study conducted over a period of six months (from July 2019 to January 2020) in two government aided secondary schools of Nandanvan area of Nagpur city, Maharashtra. Nandanvan, the urban field practice area of AIIMS Nagpur, is located in eastern part of Nagpur city, covering around 70,000 people including 27,000 slum dwellers.

Out of three identified slum areas in the study area, two schools were deliberately selected for introducing "Health Promoting School". ${ }^{10}$ Ours study was carried out as a part of baseline assessment for developing strategies for the said initiative.

\section{Study population and sample size}

All students of Class-VI to IX (10-19 years) who were present on the day of the visit and on the day of repeat visit were included in the study. Students of Standard V were excluded as many of them were $<10$ years old. Students of class-X were excluded in order not to disrupt their examination schedule. The total number of students included was 814 .

\section{Study tools and techniques}

A pre-designed and pre-tested self-administered questionnaire was administered to students in their local language comprising of background characteristics, history of hypertension amongst parents, selected risk behaviour in relation to diet, physical activity, tobacco use and their mental health status. The diet, behaviour and mental health related questions were adopted from Global School Health Survey Questionnaire. ${ }^{11}$ Anthropometric and blood pressure measurements were undertaken after explaining the procedure to the children, using standard calibrated equipment and methodology. Seca electronic scale (to the nearest $100 \mathrm{~g}$ ) was used for weight measurement. Height was measured using a stature meter $(200 \mathrm{~cm})$ to the nearest $0.1 \mathrm{~cm}$. Nutritional status was assessed using WHO BMI for age $\mathrm{z}$ score ${ }^{12}$ and categorized as severe thinness, thinness, normal, overweight and obesity. Later, overweight and obesity were categorized as 'overweight/obese' and the remaining into 'normal/thin' (Severe Thinness, Thinness and Normal).

Blood pressure (BP) was measured in the right arm with the subject seated and the arm extended over the table at the level of heart; by using a standard oscillometric and auscultatory sphygmomanometers and using suitable cuff (covering two-thirds of the upper arm and encircling it completely without overlapping). Two different cuffs (of $12 \times 22 \mathrm{~cm} \mathrm{\&}$ $9 \times 18 \mathrm{~cm}$ size) were used for that purpose. All procedures were carried out and hypertension status was classified according to the recent guideline proposed by American Pediatric Association (APA). ${ }^{13}$

Children were considered to have hypertension and prehypertension if the systolic or diastolic BP or both were $\geq 95$ th centile and $\geq 90$ th but $<95$ th centile respectively for height for age and sex as defined by APA. For students with BP of $>90$ th centile on the first reading, $\mathrm{BP}$ measurement was repeated two more times, and an average of the later readings were considered for further diagnosis. Those with high BP ( $>90$ th centile) in average readings were visited after 14 days and same procedure was repeated using aneroid sphygmomanometer. (Fig-1).

The data were collected convenient to school schedules. Each classes was divided into four sections comprising of 30 students. One section was approached each day. Those students who were not present on the day of the visit were approached the next day. Absentees on both the

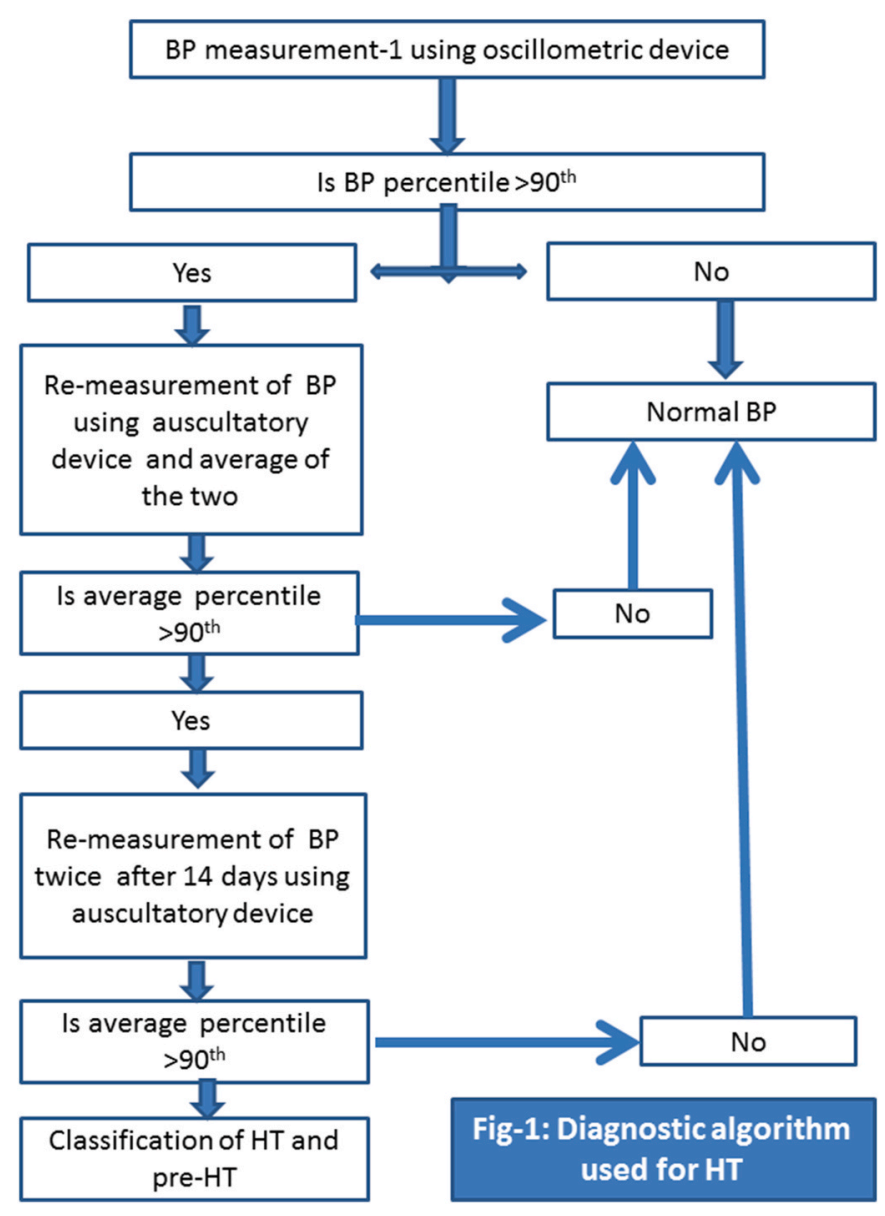

Fig. 1. Diagnostic Algorithm used for HT.

days were excluded from the study.

\section{Operational definitions}

Low fruit and vegetable intake was defined as consuming less than minimum recommended 5 servings per day (fruit and vegetable together). ${ }^{14,15}$ Data on different types of ultra-processed food consumption were recorded in using consumption of the following three items namely carbonated beverage, fast food and high sugar containing food from market. Later, if the adolescent has consumed any one of these following these three items in the last seven days it was considered as consumption of ultra processed food. Physical activity related data were collected on which type of physical activity they were involved and for how many days a week. Not performing moderate/vigorous physical activity at least five times per week (60 min per day) in the last week was categorized as inadequate physical activity. ${ }^{16}$ Students who remain four daily hours or more at the computer, television or video games on weekdays and weekends were included in the sedentary behaviour category. ${ }^{17}$ Consuming any form of tobacco product in the last 30 days was considered as 'tobacco users' ${ }^{18}$ Data on self-reported mental health symptoms were collected in five point likert scale, and those reporting presence of the symptom 'most of the times'/'always' were categorized as having the symptom. Five mental health symptoms included in the questionnaire were added and number of symptoms present in each individual was included as an independent variable during regression analysis. The presence of hypertension among any parent was considered as positive family history.

Statistical analysis: Data were entered into EpiData entry V3.1. Proportions of pre-hypertension, hypertension and health risk behaviours were expressed as number and percentage. BMI z score calculation 
and categorization was carried out by WHO Anthroplus software. Age, sex and height adjusted BP centile calculation and categorization was carried out online link for Merck Manual medical calculator. ${ }^{19,20}$ Multivariable logistic regression was run using IBM SPSS software 19.0 (Statistical Package for the Social Sciences Inc, Chicago, IL, USA), for determinants of hypertension, where all the background characteristics, behavioral risk factors, mental health and nutritional status were included in the model by forced entry method.

\section{Ethical issues}

The study was initiated after obtaining ethical clearance from Institute Ethics Committee and necessary permission from school authority. (IEC approval no: IEC/Pharmac/07/2019, AIIMS Nagpur dt December $14,2019)$ Research was carried out without disturbing routine school activities. Since the study participants were aged $>7$ and, $<18$ years, informed ascent from the participants and consent from their legal guardians were obtained. Students who were diagnosed as having high blood pressure and other health ailments were referred to the Urban Health and Training Centre. The findings in relation to risk behaviours were discussed with school teachers and modules for health promoting school initiative have been developed based on it. The researchers are now implementing Behaviour Change Interventions based on this module.

\section{Results}

Of the 910 students enrolled in the selected class 814 were included in the study (Response rate $-89.5 \%$ ). Among the 814 students, complete assessment for outcome was obtained from 774 (95.1\%) participants.

\section{Socio demographic characteristics}

The mean (SD) age of the participants was 12.88 (1.2) years. Sexes were almost equally represented (boys $51.8 \%$, girls $48.2 \%$ ) and most of the participants were Hindus (66.9\%). Median (IQR) years of schooling for fathers and mothers were 10 (3) and 10 (2) years respectively. Fathers of most of the participants were unskilled workers (49.9) and mothers were home makers (72.6\%). Around 20\% participants had history of hypertension in family.

\section{Behavioral risk factors}

The proportion of low fruit and vegetable intake and consumption of ultra-processed food was very high (85.8\% and $97.3 \%$ respectively). Proportion of participants consuming carbonated beverage, fast food and high sugar food were $69.6 \%, 90.5 \%$ and $82.3 \%$ respectively. Inadequate physical activity, sedentary lifestyle, and tobacco consumption were reported in $62.3 \%, 22.7 \%$ and $9.8 \%$ participants respectively. Overall $4.1 \%$ participants were found to be overweight/obese. Other parameters are tabulated in Table- 1 .

\section{Variations in blood pressure}

Among the 774 study participants who had their final outcome recorded, $82(10.6 \%)$ and $100(12.9 \%)$ were found to have prehypertension and hypertension respectively. Thus, total of $23.5 \%$ students diagnosed to have high blood pressure. Proportion of diastolic hypertension was higher than systolic (9.4\% vs $7.8 \%$ ) (Fig-2) [Insert Fig. 1.].Mean SBP and DBP were significantly higher in females, overweight/Obese students and those with history of poor Fruit and vegetable intake compared to their corresponding categories. Further, mean SBP was higher in those with family history of hypertension and feeling lonely most of the times. (Table-2). Bivariate analysis revealed prevalence of hypertension to be higher in those with family history of hypertension (19.1\% vs $11.1 \%$ ), whose fathers are unskilled workers or
Table 1

Behavioral and other Risk factors of hypertension by gender among the study subjects $(\mathrm{n}=774)$.

\begin{tabular}{|c|c|c|c|c|}
\hline \multirow[t]{2}{*}{ Risk factors } & & \multicolumn{3}{|c|}{ Number (\%) } \\
\hline & & $\begin{array}{l}\text { Boys (n } \\
=401)\end{array}$ & $\begin{array}{l}\text { Girls (n } \\
=373)\end{array}$ & $\begin{array}{l}\text { Total (n } \\
=774)\end{array}$ \\
\hline \multirow[t]{5}{*}{ Fruit intake in last 7 days } & No & $\begin{array}{l}63 \\
(15.7)\end{array}$ & $\begin{array}{l}43 \\
(11.5)\end{array}$ & $\begin{array}{l}106 \\
(13.7)\end{array}$ \\
\hline & Irregular (not & 148 & 165 & 313 \\
\hline & daily) & $(36.9)$ & $(44.2)$ & $(40.4)$ \\
\hline & Daily & 190 & 165 & 355 \\
\hline & & $(47.4)$ & $(44.2)$ & $(45.9)$ \\
\hline \multirow{4}{*}{$\begin{array}{l}\text { Vegetable intake in last } 7 \\
\text { days }\end{array}$} & No & $6(1.5)$ & $7(1.9)$ & $13(1.7)$ \\
\hline & $\begin{array}{l}\text { Irregular (not } \\
\text { daily) }\end{array}$ & $\begin{array}{l}53 \\
(13.2)\end{array}$ & $67(18)$ & $\begin{array}{l}120 \\
(15.5)\end{array}$ \\
\hline & Once Daily & $\begin{array}{l}120 \\
(29.9)\end{array}$ & $\begin{array}{l}110 \\
(29.5)\end{array}$ & $\begin{array}{l}230 \\
(29.7)\end{array}$ \\
\hline & $\begin{array}{l}\text { Two or more } \\
\text { times daily }\end{array}$ & $\begin{array}{l}222 \\
(55.4)\end{array}$ & $\begin{array}{l}189 \\
(50.7)\end{array}$ & $\begin{array}{l}411 \\
(53.1)\end{array}$ \\
\hline \multirow[t]{2}{*}{$\begin{array}{l}\text { Fruit and vegetable intake } \\
\text { (together) }{ }^{\mathrm{a}}\end{array}$} & $\begin{array}{l}\text { Less than } 5 \\
\text { servings per day }\end{array}$ & $\begin{array}{l}344 \\
(85.8)\end{array}$ & $\begin{array}{l}327 \\
(87.7)\end{array}$ & $\begin{array}{l}671 \\
(86.7)\end{array}$ \\
\hline & $\begin{array}{l}5 \text { or more } \\
\text { servings per day }\end{array}$ & $\begin{array}{l}57 \\
(14.2)\end{array}$ & $\begin{array}{l}46 \\
(12.3)\end{array}$ & $\begin{array}{l}103 \\
(13.3)\end{array}$ \\
\hline \multirow[t]{2}{*}{$\begin{array}{l}\text { Consumption of } \\
\text { carbonated beverage }^{a}\end{array}$} & Yes & $\begin{array}{l}279 \\
(69.6)\end{array}$ & $\begin{array}{l}241 \\
(64.6)\end{array}$ & $\begin{array}{l}520 \\
(67.2)\end{array}$ \\
\hline & No & $\begin{array}{l}122 \\
(30.4)\end{array}$ & $\begin{array}{l}132 \\
(35.4)\end{array}$ & $\begin{array}{l}254 \\
(32.8)\end{array}$ \\
\hline \multirow[t]{2}{*}{ Consumption of fast food ${ }^{a}$} & Yes & $\begin{array}{l}363 \\
(90.5)\end{array}$ & $\begin{array}{l}337 \\
(90.3)\end{array}$ & $74(9.6)$ \\
\hline & No & $38(9.5)$ & $36(9.7)$ & $\begin{array}{l}700 \\
(90.4)\end{array}$ \\
\hline \multirow{2}{*}{$\begin{array}{l}\text { Consumption of High } \\
\text { sugar containing food } \\
\text { from market }\end{array}$} & Yes & $\begin{array}{l}330 \\
(82.3)\end{array}$ & $\begin{array}{l}323 \\
(86.6)\end{array}$ & $\begin{array}{l}653 \\
(84.4)\end{array}$ \\
\hline & No & $\begin{array}{l}71 \\
(17.7)\end{array}$ & $\begin{array}{l}50 \\
(13.4)\end{array}$ & $\begin{array}{l}121 \\
(15.6)\end{array}$ \\
\hline \multirow[t]{2}{*}{$\begin{array}{l}\text { Consumption of ultra- } \\
\text { processed food }\end{array}$} & Yes & $\begin{array}{l}390 \\
(97.3)\end{array}$ & $\begin{array}{l}364 \\
(97.6)\end{array}$ & $\begin{array}{l}754 \\
(97.4)\end{array}$ \\
\hline & No & $11(2.7)$ & $9(2.4)$ & $20(2.6)$ \\
\hline \multirow[t]{2}{*}{ Sedentary lifestyle ${ }^{\mathrm{b}}$} & Yes & $\begin{array}{l}91 \\
(22.7)\end{array}$ & $\begin{array}{l}65 \\
(17.4)\end{array}$ & $\begin{array}{l}156 \\
(20.2)\end{array}$ \\
\hline & No & $\begin{array}{l}310 \\
(77.3)\end{array}$ & $\begin{array}{l}308 \\
(82.6)\end{array}$ & $\begin{array}{l}618 \\
(79.8)\end{array}$ \\
\hline \multirow[t]{2}{*}{ Physical exercise } & Inadequate & $\begin{array}{l}250 \\
(62.3)\end{array}$ & $\begin{array}{l}246 \\
(66.0)\end{array}$ & $\begin{array}{l}496 \\
(64.1)\end{array}$ \\
\hline & Adequate & $\begin{array}{l}151 \\
(37.7)\end{array}$ & $\begin{array}{l}127 \\
(34.0)\end{array}$ & $\begin{array}{l}278 \\
(35.9)\end{array}$ \\
\hline \multirow[t]{2}{*}{$\begin{array}{l}\text { Tobacco Intake (any } \\
\text { form) }\end{array}$} & Yes & $\begin{array}{l}54 \\
(13.5)\end{array}$ & $22(5.9)$ & $76(9.8)$ \\
\hline & No & $\begin{array}{l}347 \\
(86.5)\end{array}$ & $\begin{array}{l}351 \\
(94.1)\end{array}$ & $\begin{array}{l}698 \\
(90.2)\end{array}$ \\
\hline \multirow[t]{2}{*}{ Smoking tobacco intake } & Yes & $19(4.7)$ & $10(2.7)$ & 29 (3.7) \\
\hline & No & $\begin{array}{l}382 \\
(95.3)\end{array}$ & $\begin{array}{l}363 \\
(97.3)\end{array}$ & $\begin{array}{l}745 \\
(96.3)\end{array}$ \\
\hline \multirow[t]{2}{*}{ Smokeless tobacco intake } & Yes & $\begin{array}{l}45 \\
(11.2)\end{array}$ & $18(4.8)$ & $63(8.1)$ \\
\hline & No & $\begin{array}{l}356 \\
(88.8)\end{array}$ & $\begin{array}{l}355 \\
(95.2)\end{array}$ & $\begin{array}{l}711 \\
(91.9)\end{array}$ \\
\hline \multirow{2}{*}{$\begin{array}{l}\text { Felt lonely most of the } \\
\text { times }{ }^{* a}\end{array}$} & Yes & $39(9.7)$ & $32(8.6)$ & $71(9.2)$ \\
\hline & No & $\begin{array}{l}362 \\
(90.3)\end{array}$ & $\begin{array}{l}341 \\
(91.4)\end{array}$ & $\begin{array}{l}703 \\
(90.8)\end{array}$ \\
\hline \multirow{2}{*}{$\begin{array}{l}\text { So worried about } \\
\text { something that could } \\
\text { not sleep at night }{ }^{*^{a}}\end{array}$} & $\begin{array}{l}\text { Yes (most of the } \\
\text { times) }\end{array}$ & $30(7.5)$ & $20(5.4)$ & $50(6.5)$ \\
\hline & No & $\begin{array}{l}371 \\
(92.5)\end{array}$ & $\begin{array}{l}353 \\
(94.6)\end{array}$ & $\begin{array}{l}724 \\
(93.5)\end{array}$ \\
\hline \multirow[t]{2}{*}{ Close friend } & No & $23(5.7)$ & $26(7.0)$ & $49(6.3)$ \\
\hline & Yes $(\geq 1)$ & $\begin{array}{l}378 \\
(94.3)\end{array}$ & $\begin{array}{l}347 \\
(93.0)\end{array}$ & $\begin{array}{l}725 \\
(93.7)\end{array}$ \\
\hline \multirow[t]{2}{*}{ Depressive symptoms } & Yes & $\begin{array}{l}87 \\
(21.7)\end{array}$ & $\begin{array}{l}73 \\
(19.6)\end{array}$ & $\begin{array}{l}160 \\
(20.7)\end{array}$ \\
\hline & No & $\begin{array}{l}314 \\
(78.3)\end{array}$ & $\begin{array}{l}300 \\
(80.4)\end{array}$ & $\begin{array}{l}614 \\
(79.3)\end{array}$ \\
\hline \multirow[t]{2}{*}{ Nutritional status } & Normal/Thin & $\begin{array}{l}386 \\
(96.3)\end{array}$ & $\begin{array}{l}356 \\
(95.4)\end{array}$ & $\begin{array}{l}742 \\
(95.9)\end{array}$ \\
\hline & $\begin{array}{l}\text { Overweight/ } \\
\text { obese }\end{array}$ & $15(3.7)$ & $17(4.6)$ & $32(4.1)$ \\
\hline
\end{tabular}

Fruit and vegetable combined intake of less than 5 servings per day.

a At least once in last 7 days. 
b More than $2 \mathrm{~h}$ of screen time.

\section{Prevalence of pre-hypertension and Hypertension among the study participants $(n=774)$}

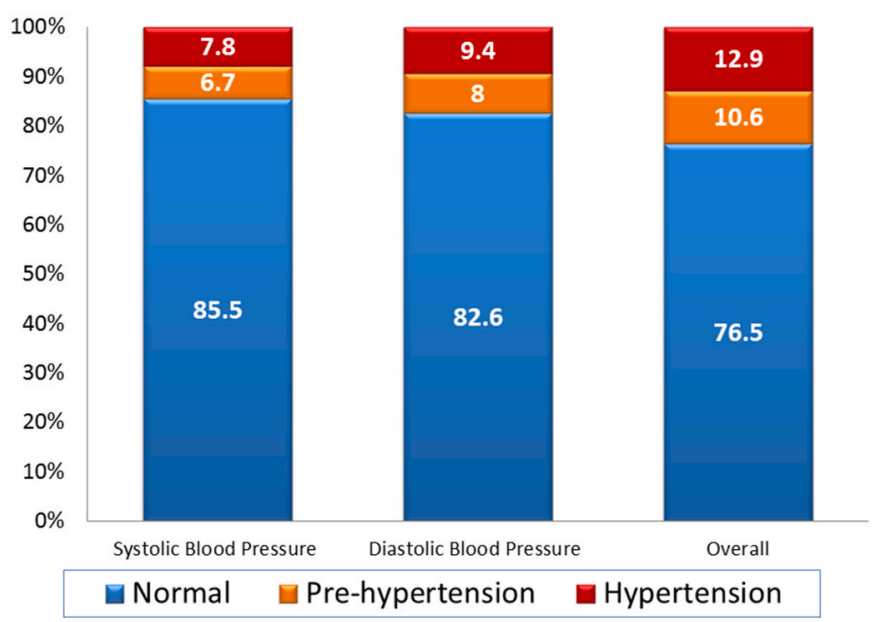

Fig. 2. Prevalence of Pre-hypertension and Hypertension among study participants $(n=774)$.

unemployed (15.5\% vs 9.4\%), overweight/obese (28.1\% vs $12.3 \%$ ), and those having mental health problems $(29.2 \%$ vs $10.8 \%)$ (Table- 3$)$. Hypertension was not found to be associated with tobacco intake and sedentary lifestyle and consumption of ultra-processed food.

Multivariable logistic regression model was significant as revealed by the omnibus Chi-square statistic, and. Good fit as evident from nonsignificant Hosmer-Lemeshow statistic. The model explained 12.9\% variance of HT. After adjusting all potential confounders, father's occupation [AOR $(96 \% \mathrm{CI}=1.89(1.06-3.41)$ ], family history of hypertension [AOR $(96 \% \mathrm{CI}=2.08(1.27-3.41)]$, number of mental health symptoms [AOR $(96 \% \mathrm{CI}=2.62(1.33-5.16)$ for 2 symptoms, 3.92 (1.40-10.96) for $\geq 3$ symptoms] and presence of overweight/obesity [AOR ( $96 \%$ CI $=3.61$ (1.53-8.53)] had significantly increased odds of having hypertension compared to others (Table-4) (Table-4).

\section{Discussion}

It is a matter of concern that nearly one quarter of school going adolescents from the identified urban slums were having high BP (prevalence of hypertension and pre-hypertension being $12.9 \%$ and $10.6 \%$ respectively). Hypertension among school going adolescents has become a matter of growing concern among researchers and a number of studies have captured this particular problem in recent years. However it is being observed that the prevalence of pre-hypertension and hypertension have wide variations across studies, even among studies from similar settings. In a study among students in a slum area of Kolkata about $30.3 \%$ students were found to be having high BP (HT- $10.1 \%$, preHT- $20.2 \%) .{ }^{21}$ Lower prevalence was observed in a recent study among adolescent boys of 9th to 12th classes from four different schools in Delhi, where prevalence of HT and pre-HT were found to be $4.3 \%$ and $7.3 \%$ respectively. ${ }^{9}$ Prevalence of hypertension as observed in our study was higher than that observed by Gupta $\mathrm{R}$ et al. in Eastern India $(7.2 \%),{ }^{22}$ Anand $\mathrm{T}$ et al. in Delhi (7\%) ${ }^{23}$ and Buch $\mathrm{N}$ et al. in Surat, $(6.48 \%) .{ }^{24}$ On the other hand, prevalence of pre-hypertension was lower (10.6\%) than what was captured by Anand T et al. in Delhi (30.1\%), ${ }^{23}$ Sharma A et al. ${ }^{12}$ in Shimla $(20 \%)^{25}$ and Kumar R et al. in Moradabad $(15.8 \%) .{ }^{26}$ Such a variablilty has been reiterated by Nayak BS et al. in a systematic review on prevalence of HT among children less than 18 years of age in South Asia where the prevalence of HT among children
Table 2

Variation of Systolic and Diastolic blood pressure among study participants based on selected baseline characteristics and risk factors $(n=774)$.

\begin{tabular}{|c|c|c|c|c|c|}
\hline Variables & Categories & $\begin{array}{l}\text { SBP } \\
\text { Mean } \\
(\mathrm{SD})\end{array}$ & $\begin{array}{l}\text { Statistics } \\
\text { (p value) }\end{array}$ & $\begin{array}{l}\text { DBP } \\
\text { Mean } \\
\text { (SD) }\end{array}$ & $\begin{array}{l}\text { Statistics } \\
\text { (p value) }\end{array}$ \\
\hline \multirow[t]{9}{*}{ Age (in Years) } & $\leq 11^{\mathrm{a}}$ & $\begin{array}{l}102.96 \\
(11.4)\end{array}$ & \multirow[t]{9}{*}{$\begin{array}{l}<\mathbf{0} / \\
\mathbf{0 0 1}^{\mathrm{a}},\end{array}$} & $\begin{array}{l}67.62 \\
(9.9)\end{array}$ & \multirow[t]{9}{*}{$0.096^{\mathrm{a}}$} \\
\hline & \multirow[t]{2}{*}{$12^{\mathrm{b}}$} & 106.6 & & 68.2 & \\
\hline & & (10.3) & & $(7.3)$ & \\
\hline & \multirow[t]{2}{*}{$13^{\mathrm{c}}$} & 110.36 & & 69.81 & \\
\hline & & (10.9) & & $(10.6)$ & \\
\hline & \multirow[t]{2}{*}{$14^{\mathrm{d}}$} & 110.8 & & 69.4 & \\
\hline & & (12.6) & & $(9.7)$ & \\
\hline & \multirow[t]{2}{*}{$\geq 15^{\mathrm{e}}$} & 112.1 & & 70.55 & \\
\hline & & (11.3) & & $(9.1)$ & \\
\hline \multirow[t]{4}{*}{ Sex } & \multirow[t]{2}{*}{ Male } & 107.62 & \multirow[t]{4}{*}{0.012} & 68.29 & \multirow[t]{4}{*}{0.019} \\
\hline & & (11.76) & & $(9.9)$ & \\
\hline & \multirow[t]{2}{*}{ Female } & 109.71 & & 69.85 & \\
\hline & & (11.18) & & (8.49) & \\
\hline \multirow{4}{*}{$\begin{array}{l}\text { Family history } \\
\text { of } \\
\text { hypertension }\end{array}$} & \multirow[t]{2}{*}{ No } & 107.9 & \multirow[t]{4}{*}{0.01} & 68.8 & \multirow[t]{4}{*}{0.27} \\
\hline & & (11.4) & & (8.9) & \\
\hline & \multirow[t]{2}{*}{ Yes } & 111.1 & & 69.7 & \\
\hline & & (11.7) & & (10.3) & \\
\hline Nutritional & Normal/thin & 108.37 & 0.004 & 68.85 & 0.006 \\
\hline status & & (11.43) & & (9.19) & \\
\hline & Overweight/ & 114.44 & & 73.44 & \\
\hline & Obese & (12.43) & & (10.42) & \\
\hline Poor Fruit and & No & 105.5 & 0.003 & 66.9 & 0.015 \\
\hline vegetable & & (10.7) & & (9.1) & \\
\hline intake & Yes & 109.1 & & 69.4 & \\
\hline & & $(11.6)$ & & $(9.3)$ & \\
\hline Consumption & No & 112.1 & 0.167 & 73.7 & 0.064 \\
\hline of ultra & & $(9.1)$ & & (10.9) & \\
\hline processed & Yes & 108.5 & & 68.9 & \\
\hline food & & (11.6) & & $(9.2)$ & \\
\hline Sedentary & No & 108.7 & 0.80 & 69.09 & 0.75 \\
\hline lifestyle & & (11.7) & & $(9.2)$ & \\
\hline & Yes & 108.4 & & 68.8 & \\
\hline & & (10.7) & & $(9.8)$ & \\
\hline Inadequate & No & 109.0 & 0.46 & 69.41 & 0.14 \\
\hline physical & & (10.9) & & $(8.9)$ & \\
\hline activity & Yes & 108.4 & & 68.38 & \\
\hline & & (11.9) & & $(9.7)$ & \\
\hline Tobacco & No & 108.8 & 0.37 & 69.2 & 0.24 \\
\hline consumption & & $(11.6)$ & & (9.3) & \\
\hline & Yes & 107.5 & & 67.8 & \\
\hline & & $(10.2)$ & & $(9.0)$ & \\
\hline Feeing lonely & No & 108.3 & 0.017 & 68.6 & 0.067 \\
\hline most of the & & $(11.2)$ & & $(9.2)$ & \\
\hline times & Yes & 112.3 & & 70.9 & \\
\hline & & (13.6) & & $(10.2)$ & \\
\hline Depressive & No & 108.5 & 0.68 & 68.9 & 0.58 \\
\hline symptom & & (11.5) & & (9.3) & \\
\hline & Yes & 108.9 & & 69.4 & \\
\hline & & (11.6) & & (8.9) & \\
\hline Sleep & No & 108.7 & 0.54 & 69.1 & 0.22 \\
\hline disturbance & & (11.3) & & $(9.2)$ & \\
\hline & Yes & 107.7 & & 67.5 & \\
\hline & & (14.2) & & (10.5) & \\
\hline Close friend & At least one & 108.7 & 0.49 & 69.1 & 0.11 \\
\hline & & (11.4) & & (9.1) & \\
\hline & No & 107.6 & & 67.0 & \\
\hline & & (13.1) & & (10.3) & \\
\hline
\end{tabular}

@Independent sample $t$-test used.

a One-way ANOVA used.

b Post-hoc test revealed significant difference between a-c, a-d, a-e, b-c, b$\mathrm{d}$ and b-e.

ranged from $0.02 \%$ to $11.7 \%$ in different studies fromNorth India (and from $0.6 \%$ to $25.6 \%$ in studies from South India. ${ }^{27}$ The probable reasons behind such variations are difference in norms of measurement, geographic factor and inclusion of different age categories (early vs late adolescents). ${ }^{27}$

We found fewer to be overweight/obese (only 4.1\%), the value being lower than what was observed in similar studies. ${ }^{28,29}$ The reason may be 
Table 3

Factors associated with hypertension among the study participants: Bivariate analysis $(n=774)$.

\begin{tabular}{|c|c|c|c|}
\hline Factors & Categories & $\begin{array}{l}\text { Hypertension } \\
\text { Number (\%) }\end{array}$ & OR $(95 \% \mathrm{CI})$ \\
\hline \multirow[t]{5}{*}{ Age (in Years) } & $\leq 11(\mathrm{n}=85)$ & $12(14.1)$ & $\begin{array}{l}1.97 \\
(0.7-5.54)\end{array}$ \\
\hline & $12(\mathrm{n}=243)$ & $25(10.3)$ & $\begin{array}{l}1.38 \\
(0.54-3.49)\end{array}$ \\
\hline & $13(\mathrm{n}=207)$ & $32(15.5)$ & $\begin{array}{l}2.19 \\
(0.88-5.47)\end{array}$ \\
\hline & $14(\mathrm{n}=161)$ & $25(15.5)$ & $\begin{array}{l}2.21 \\
(0.87-5.62)\end{array}$ \\
\hline & $\geq 15(\mathrm{n}=78)$ & $6(7.7)$ & 1 \\
\hline \multirow[t]{2}{*}{ Sex } & Female $(\mathrm{n}=373)$ & $51(13.7)$ & $\begin{array}{l}1.14 \\
(0.75-1.73)\end{array}$ \\
\hline & Male $(\mathrm{n}=401)$ & $49(12.2)$ & 1 \\
\hline \multirow[t]{3}{*}{ Mother's education } & $\begin{array}{l}\text { Non-formal, Primary } \\
\text { and middle }(\mathrm{n}=287)\end{array}$ & $40(13.9)$ & $\begin{array}{l}1.28 \\
(0.69-3.37)\end{array}$ \\
\hline & Secondary $(\mathrm{n}=343)$ & $44(12.8)$ & $\begin{array}{l}1.16 \\
(0.63-2.13)\end{array}$ \\
\hline & HS and above (142) & $16(11.3)$ & 1 \\
\hline \multirow[t]{4}{*}{ Mother's occupation } & $\begin{array}{l}\text { Unskilled private job ( } \mathrm{n} \\
=65 \text { ) }\end{array}$ & $13(20.0)$ & $\begin{array}{l}2.94 \\
(0.89-9.64)\end{array}$ \\
\hline & Maid servant $(\mathrm{n}=94)$ & $15(16.0)$ & $\begin{array}{l}2.23 \\
(0.69-7.12)\end{array}$ \\
\hline & Home maker $(\mathrm{n}=562)$ & $68(12.1)$ & $\begin{array}{l}1.62 \\
(0.57-4.63)\end{array}$ \\
\hline & $\begin{array}{l}\text { Self-employed/skilled } \\
\text { workers }(n=51)\end{array}$ & $4(7.8)$ & 1 \\
\hline \multirow[t]{4}{*}{ Father's education } & $\begin{array}{l}\text { Non-formal and below } \\
\text { primary }(n=46)\end{array}$ & $7(15.2)$ & $\begin{array}{l}1.25 \\
(0.49-3.18)\end{array}$ \\
\hline & $\begin{array}{l}\text { Primary and middle ( } \mathrm{n} \\
=245 \text { ) }\end{array}$ & $22(9.0)$ & $\begin{array}{l}0.68 \\
(0.36-1.31)\end{array}$ \\
\hline & Secondary $(n=328)$ & $52(15.9)$ & $\begin{array}{l}1.31 \\
(0.74-2.30)\end{array}$ \\
\hline & HS and above $(\mathrm{n}=151)$ & $19(12.6)$ & 1 \\
\hline \multirow[t]{3}{*}{ Father's occupation } & $\begin{array}{l}\text { Unskilled workers and } \\
\text { unemployed }(n=400)\end{array}$ & $62(15.5)$ & $\begin{array}{l}1.76 \\
(1.03-3.00)\end{array}$ \\
\hline & $\begin{array}{l}\text { Semi-skilled workers ( } \mathrm{n} \\
=158 \text { ) }\end{array}$ & $18(11.4)$ & $\begin{array}{l}1.23 \\
(0.63-2.42)\end{array}$ \\
\hline & $\begin{array}{l}\text { Skilled worker and } \\
\text { semi-professional }(\mathrm{n}= \\
\text { 212) }\end{array}$ & $20(9.4)$ & 1 \\
\hline \multirow[t]{2}{*}{$\begin{array}{l}\text { Family } \mathrm{H} / \mathrm{O} \\
\text { Hypertension }\end{array}$} & Yes $(\mathrm{n}=173)$ & $33(19.1)$ & $\begin{array}{l}1.88 \\
(1.19-2.97)\end{array}$ \\
\hline & No $(n=601)$ & $67(11.1)$ & 1 \\
\hline \multirow[t]{2}{*}{$\begin{array}{l}\text { Poor Fruit and } \\
\text { vegetable intake }\end{array}$} & Yes $(\mathrm{n}=671)$ & $90(13.4)$ & $\begin{array}{l}1.44 \\
(0.72-2.87)\end{array}$ \\
\hline & No $(n=103)$ & $10(9.7)$ & 1 \\
\hline \multirow[t]{2}{*}{$\begin{array}{l}\text { Consumption of ultra } \\
\text { processed food }\end{array}$} & Yes $(\mathrm{n}=754)$ & $96(12.7)$ & $\begin{array}{l}0.34 \\
(0.19-1.78)\end{array}$ \\
\hline & No $(n=20)$ & $4(20.0)$ & 1 \\
\hline \multirow[t]{2}{*}{ Sedentary lifestyle } & Yes $(n=156)$ & $21(13.5)$ & $\begin{array}{l}1.06 \\
(0.63-1.78)\end{array}$ \\
\hline & No $(n=618)$ & $79(12.8)$ & 1 \\
\hline \multirow[t]{2}{*}{$\begin{array}{l}\text { Inadequate physical } \\
\text { activity }\end{array}$} & Yes $(n=496)$ & $61(12.3)$ & $\begin{array}{l}0.86 \\
(0.56-1.32)\end{array}$ \\
\hline & No $(n=278)$ & $39(14.0)$ & 1 \\
\hline \multirow[t]{2}{*}{$\begin{array}{l}\text { Tobacco } \\
\text { consumption }\end{array}$} & Yes $(\mathrm{n}=76)$ & $7(9.2)$ & $\begin{array}{l}0.66 \\
(0.29-1.48)\end{array}$ \\
\hline & No $(n=698)$ & $93(13.3)$ & 1 \\
\hline \multirow[t]{4}{*}{$\begin{array}{l}\text { Mental Health } \\
\text { problems }\end{array}$} & $\geq 3(n=24)$ & $7(29.2)$ & $\begin{array}{l}3.40 \\
(1.35-8.58)\end{array}$ \\
\hline & $2(\mathrm{n}=73)$ & $16(21.9)$ & $\begin{array}{l}2.32 \\
(1.24-4.33)\end{array}$ \\
\hline & $1(\mathrm{n}=186)$ & 24 (12.9) & $\begin{array}{l}1.22 \\
(0.73-2.05)\end{array}$ \\
\hline & $\operatorname{Nil}(n=491)$ & $53(10.8)$ & 1 \\
\hline \multirow[t]{2}{*}{ Nutritional status } & $\begin{array}{l}\text { Overweight/Obese ( } \mathrm{n}= \\
\text { 32) }\end{array}$ & $9(28.1)$ & $\begin{array}{l}2.79 \\
(1.25-6.24)\end{array}$ \\
\hline & Normal/thin $(\mathrm{n}=742)$ & $91(12.3)$ & 1 \\
\hline
\end{tabular}

Table 4

Factors associated with hypertension among the study participants: multivariable logistic regression $(n=774)$.

\begin{tabular}{|c|c|c|}
\hline Variables & Categories & AOR $(95 \% \mathrm{CI})$ \\
\hline Constant & & 0.044 \\
\hline \multirow{4}{*}{$\begin{array}{l}\text { Age (in Years) } \\
\quad \text { (Ref: } \leq 11)\end{array}$} & 12 & $\begin{array}{l}0.71 \\
(0.32-1.56)\end{array}$ \\
\hline & 13 & $\begin{array}{l}1.20 \\
(0.54-2.67)\end{array}$ \\
\hline & 14 & $\begin{array}{l}1.21 \\
(0.53-2.74)\end{array}$ \\
\hline & $\geq 15$ & $\begin{array}{l}0.44 \\
(0.15-1.36)\end{array}$ \\
\hline Sex (Ref: Male) & Female & $\begin{array}{l}1.02 \\
(0.65-1.60)\end{array}$ \\
\hline $\begin{array}{l}\text { Mother's education (Ref: HS and } \\
\text { above) }\end{array}$ & $\begin{array}{l}\text { Primary and middle } \\
\text { school } \\
\text { Secondary }\end{array}$ & $\begin{array}{l}1.66 \\
(0.84-3.28) \\
1.26 \\
(0.66-2.41)\end{array}$ \\
\hline \multirow[t]{3}{*}{$\begin{array}{l}\text { Mother's occupation (Ref: Self- } \\
\text { employed/skilled workers) }\end{array}$} & Unskilled private job & $\begin{array}{l}2.57 \\
(0.74-8.94)\end{array}$ \\
\hline & Maid servant & $\begin{array}{l}2.09 \\
(0.61-7.19)\end{array}$ \\
\hline & Home maker & $\begin{array}{l}1.74 \\
(0.58-5.29)\end{array}$ \\
\hline \multirow[t]{3}{*}{$\begin{array}{l}\text { Father's education (Ref: HS and } \\
\text { above) }\end{array}$} & Below primary & $\begin{array}{l}1.01 \\
(0.36-2.78)\end{array}$ \\
\hline & Primary and middle & $\begin{array}{l}0.53 \\
(0.25-1.08)\end{array}$ \\
\hline & Secondary & $\begin{array}{l}1.37 \\
(0.74-2.53)\end{array}$ \\
\hline $\begin{array}{l}\text { Father's occupation (Ref: Skilled } \\
\text { worker \& semi-professional) }\end{array}$ & $\begin{array}{l}\text { Unskilled workers and } \\
\text { unemployed }\end{array}$ & $\begin{array}{l}1.89 \\
(1.06-3.41)\end{array}$ \\
\hline & Semi-skilled workers & $\begin{array}{l}1.31 \\
(0.64-2.68)\end{array}$ \\
\hline $\begin{array}{l}\text { Family history of Hypertension (Ref: } \\
\text { No) }\end{array}$ & Yes & $\begin{array}{l}2.08 \\
(1.27-3.41)\end{array}$ \\
\hline $\begin{array}{l}\text { Fruit and vegetable intake (Ref: } \\
\text { Adequate) }\end{array}$ & Poor & $\begin{array}{l}1.40 \\
(0.67-2.93)\end{array}$ \\
\hline $\begin{array}{l}\text { Consumption of ultra processed food } \\
\text { (Ref: No) }\end{array}$ & Yes & $\begin{array}{l}0.57 \\
(0.18-1.87)\end{array}$ \\
\hline Sedentary lifestyle (Ref: No) & Yes & $\begin{array}{l}0.95 \\
(0.54-1.67)\end{array}$ \\
\hline Physical activity (Ref: Adequate) & Inadequate & $\begin{array}{l}0.74 \\
(0.46-1.17)\end{array}$ \\
\hline Tobacco consumption (Ref: No) & Yes & $\begin{array}{l}0.56 \\
(0.24-1.35)\end{array}$ \\
\hline \multirow[t]{3}{*}{ Mental Health problems (Ref: Nil) } & $\geq 3$ & $\begin{array}{l}3.92 \\
(1.40-10.96)\end{array}$ \\
\hline & 2 & $\begin{array}{l}2.62 \\
(1.33-5.16)\end{array}$ \\
\hline & 1 & $\begin{array}{l}1.21 \\
(0.70-2.09)\end{array}$ \\
\hline $\begin{array}{l}\text { Nutritional status: (Ref: Normal/ } \\
\text { thin) }\end{array}$ & Overweight/Obese & $\begin{array}{l}3.61 \\
(1.53-8.53)\end{array}$ \\
\hline Model significance & & 0.006 \\
\hline Nagelkarke $\mathrm{R}^{2}$ & & 0.129 \\
\hline Hosmer and Lemeshow Test & & 0.493 \\
\hline
\end{tabular}

inclusion of only slum students in this study who are generally underweight than their non-slum counterparts. We observed a significant association between hypertension and elevated BMI, as found elsewhere." $2530{ }^{31}$ Here hypertension was found to be significantly associated with symptoms of mental ill-health. Ojike $\mathrm{N}$ et al. (2016) analyzed National Health Interview Survey for 2004-2013 data for US population and observed psychological distress to be associated with higher odds of hypertension after adjusting for other risk factors. ${ }^{32}$ Psychological factors were reported as important etiological drivers for the development of hypertension by others too ${ }^{33,34}$

No association was found between hypertension and with physical activity, the result being in line with a similar study among adolescent boys in Delhi. ${ }^{9}$ In contrast Christofaro et al.(2011) observed an inverse correlation between total physical activity and blood pressure among Brazilian adolescents. ${ }^{35}$ 
In this study, both mean SBP and DBP were significantly high in students consuming inadequate Fruits and vegetables. Significant difference in fruit and vegetable intake among normotensives and prehypertensives was also found elsewhere. ${ }^{9}$ and also supported by the finding that increase in consumption of fruits and vegetables from $<3$ servings/day to $>5$ servings/day resulted in $17 \%$ reduction in $\mathrm{CHD}$ risk. ${ }^{36}$

In this study prevalence of tobacco use was found to be $9.8 \%$. In a recent systematic review, Raja B K et al. (2018) reported prevalence of tobacco use among school going adolescents in India involving 20 published articles and found wide variation of tobacco use $(5.9 \%-$ $49 \%) .{ }^{37}$ on The recent round of Global Adult Tobacco Survey-2 had found $12.2 \%$ smokers using tobacco daily before the age of 15 years. ${ }^{38}$ Major strength of the study was that the measurement of blood pressure was done with methodological vigour. . Firstly, we used validated methods for anthropometry and BP measurement and classified the status of hypertension as appropriate for adolescent age group. Secondly, we chose appropriate cuff size as per the age group and arm circumference. Thirdly, this study labels the status as hypertension after confirming the high Blood pressure in three phases using validated aneroid apparatus. Hence, hypertension identified in this study is unlikely to have a false positive error or misdiagnosis. Fourth, for this study all BP measurements were assessed by single trained community care physician. Thus, the common problem of inter observation variation in BP measurement is being obviated. Further, response rate of the study population was high (89.5\%).

However, the findings of this study should be interpreted with limited external validity, as students of only two schools located in slum areas are included in it, and students were mostly from lower socioeconomic status. Certain risk factors of hypertension like salt intake, kidney disease, presence of other NCD (Diabetes, high cholesterol) etc that might have association with BP could not be included. Moreover seven day recall was used for assessing dietary pattern, so chance of recall bias cannot be ruled out. As there is no existing standards specific for Indian adolescents, widely used APA standards were used. ${ }^{12}$ The possibility of the estimates i.e. prevalence of hypertension and prehypertension being grossly influenced by country or ethnicity-specific standards warrants further exploration.

As consumption of ultra-processed food was very high, and considerable number of children were consuming tobacco, school should develop health promoting policy and strictly implement existing government policy to restrict advertisement, promotion, and availability of tobacco products and ultra-processed foods in and around the school premises. Schools should be strictly made tobacco free zone. It was very unfortunate that involvement in physical activity was inadequate among a large number of students. Moreover spending long duration in front of screen was also common among them. Urgent attention should be given for promotion of outdoor sports, game etc. At school settings. Moreover high proportion of mental health issues, and its positive association with HT imposes the need to actively screen students with suspected mental health symptoms by school teachers. School should involve students in recreational activities, yoga and arrange de-stressing sessions for at risk students.

\section{Conclusion}

As the prevalence of HT and its risk factors are very high amongst school children, it emphasize the need for delivering focused intervention with special attention on largely preventable behavioral and mental health issues. Implementing these 'Best Buys' strategy ${ }^{39}$ will avert more number of hypertension and adverse consequence in future. This also indicates the need for periodic monitoring of BP and anthropometry among school children which could be planned during annual school health medical examination. If students are identified 'at risk' they have to be linked for treatment or tracked for future development of Hypertension as applicable.

\section{Funding}

Self-funded.

\section{Declaration of competing interest}

None.

\section{Acknowledgement}

We acknowledge all the students and teachers who helped us in carrying out the research work.

\section{References}

1 Yadav S, Arokiasamy P. Understanding Epidemiological Transition in India. Glob Health Action [Internet]; 2014 May 15, 7. [cited 2021 Mar 19] Available from: http://www, ncbi.nlm.nih.gov/pmc/articles/PMC4028906/.

2 Gupta R, Xavier D. Hypertension: the most important non communicable disease risk factor in India. Indian Heart J. 2018 Jul 1;70(4):565-572.

3 Tackling G, Borhade MB. Hypertensive heart disease. In: StatPearls [Internet]. Treasure Island (FL). StatPearls Publishing; 2020 [cited 2021 Mar 19] Available from: http://www.ncbi.nlm.nih.gov/books/NBK539800/.

4 Sabri M, Gheissari A, Mansourian M, Mohammadifard N, Sarrafzadegan N. Essential hypertension in children, a growing worldwide problem. J Res Med Sci Off J Isfahan Univ Med Sci [Internet; 2019 Dec 23, 24. [cited 2021 Mar 19] Available from: http s://www.ncbi.nlm.nih.gov/pmc/articles/PMC6950331/.

5 Brady TM. Obesity-related hypertension in children. In: Front Pediatr [Internet]; 2017 Sep 25, 5. Available from: http://www.ncbi.nlm.nih.gov/pmc/articles/PM C5622310/.

6 Mucci N, Giorgi G, De Pasquale Ceratti S, Fiz-Pérez J, Mucci F, Arcangeli G. Anxiety, Stress-Related Factors, and Blood Pressure in Young Adults. Front Psychol [Internet]; 2016 Oct 28, 7. [cited 2021 Mar 19] Available from: http://www.ncbi.nlm.nih. gov/pmc/articles/PMC5083786/.

7 Luma GB, Spiotta RT. Hypertension in children and adolescents. Am Fam Physician. 2006 May 1;73(9):1558-1566.

8 Anyaegbu E, Dharnidharka V. Hypertension IN the teenager. Pediatr Clin. 2014 Feb; 61(1):131-151.

9 Singh SK, Verma A. Prevalence of hypertension among school going adolescent boys in Najafgarh, Delhi, India. Int J Adolesc Med Health. 2020 Jan 18.

10 Health promoting schools [Internet]. [cited 2021 Mar 19]. Available from: http s://www.who.int/westernpacific/health-topics/health-promoting-schools.

11 Questionnaire - CDC Global School-based Student Health Survey (GSHS) [Internet]. 2019 [cited 2021 Mar 19]. Available from: https://www.cdc.gov/gshs/ques tionnaire/index.htm.

12 WHO | BMI-for-age (5-19 years) [Internet]. WHO [cited 2021 Mar 19]. Available from: http://www.who.int/growthref/who2007_bmi_for_age/en/.

13 Flynn JT, Kaelber DC, Baker-Smith CM, et al. Clinical Practice Guideline for Screening and Management of High Blood Pressure in Children and Adolescents. Pediatrics [Internet]; 2017 Sep 1 [cited 2021 Mar 19];140(3). Available from: https://pediat rics.aappublications.org/content/140/3/e20171904.

14 Adolescents. health-related behaviours - Nutrition [Internet]. [cited 2021 Mar 19]. Available from: https://apps.who.int/adolescent/second-decade/section4/page4/ Nutrition.html.

15 Healthy diet [Internet]. [cited 2021 Mar 19]. Available from: https://www.who.int/ news-room/fact-sheets/detail/healthy-diet.

16 How Much Physical Activity Do Children Need?. Physical Activity | CDC [Internet]. 2020 [cited 2021 Mar 19]. Available from: https://www.cdc.gov/physicalactivity/ basics/children/index.htm.

17 Moraes ACF de, Fernandes CAM, Elias RGM, Nakashima ATA, Reichert FF, Falcão MC. [Prevalence of physical inactivity and associated factors in adolescents]. Rev Assoc Med Bras. 2009 Oct;55(5):523-528.

18 Nunes HEG, Gonçalves EC de A, Vieira JAJ, Silva DAS. Clustering of risk factors for non-communicable diseases among adolescents from southern Brazil [Internet] PloS One. 2016 Jul 19;11(7) [cited 2021 Mar 19] Available from: http://www.ncbi.nlm. nih.gov/pmc/articles/PMC4951139/.

19 Blood Pressure Percentiles for Boys (2 - 17 years) [Internet]. [cited 2021 Mar 19]. Available from: https://www.merckmanuals.com/medical-calculators/BloodPress urePercentBoys.htm.

20 Blood Pressure Percentiles for Girls (2 - 17 years) [Internet]. [cited 2021 Mar 19]. Available from: https://www.merckmanuals.com/medical-calculators/BloodPress urePercentGirls.htm.

21 Maiti M, Bandyopadhyay L. Variation in blood pressure among adolescent schoolchildren in an urban slum of Kolkata, West Bengal. Postgrad Med. 2017 Nov;93 (1105):648-652.

22 Gupta R, Goyle A, Kashyap S, Agarwal M, Consul R, Jain BK. Prevalence of atherosclerosis risk factors in adolescent school children. Indian Heart J. 1998 Oct;50 (5):511-515.

23 Anand T, Ingle GK, Meena GS, Kishore J, Kumar R. Hypertension and its correlates among school adolescents in Delhi. Int J Prev Med. 2014 Mar;5(Suppl 1):S65-S70. 
24 Buch N, Goyal JP, Kumar N, Parmar I, Shah VB, Charan J. Prevalence of hypertension in school going children of Surat city, Western India. J Cardiovasc Dis Res. 2011;2(4): 228-232.

25 Sharma A, Grover N, Kaushik S, Bhardwaj R, Sankhyan N. Prevalence of hypertension among schoolchildren in Shimla. Indian Pediatr. 2010 Oct;47(10): 873-876.

26 Kumar R, Sharma M, Srivastava A. A cross sectional study of hypertension in adolescent girls of district Moradabad, Uttar Pradesh, India. Int J Community Med Public Health. 2017 Jan 5;3(6):1388-1392.

27 Nayak BS, Dsouza A, Shetty S, Ravishankar N. Prevalence of childhood hypertension in South Asia: a systematic review and meta-analysis [Internet] J Clin Diagn Res; 2018 [cited 2021 Mar 19] Available from: https://jcdr.net/article_fulltext.asp?iss $\mathrm{n}=0973-709 \mathrm{x}$ \&year $=2018$ \&volume $=12$ \&issue $=10$ \&page $=$ LC13\&issn $=0973-709 \mathrm{x}$ $\&$ id $=12200$.

28 Gupta D, Shah P, Misra A, et al. Secular trends in prevalence of overweight and obesity from 2006 to 2009 in urban asian Indian adolescents aged 14-17 years. PloS One. 2011 Feb 23;6, e17221.

29 Ghonge S, Adhav PS, Landge J, Thakor N. Prevalence of obesity and overweight among school children of Pune city, Maharashtra, India: a cross sectional study. Int $J$ Res Med Sci. 2017 Jan 17;3(12):3599-3603.

30 Durrani AM, Fatima W. Determinants of blood pressure distribution in school children. Eur J Publ Health. 2012 Jun;22(3):369-373.

31 Taksande A, Chaturvedi P, Vilhekar K, Jain M. Distribution of blood pressure in school going children in rural area of Wardha district, Maharashatra, India. Ann Pediatr Cardiol. 2008 Jul;1(2):101-106.

32 Ojike N, Sowers JR, Seixas A, et al. Psychological distress and hypertension: results from the national health Interview Survey for 2004-2013. Cardiorenal Med. 2016;6 (3):198-208.
33 Footman K, Roberts B, Tumanov S, McKee M. The comorbidity of hypertension and psychological distress: a study of nine countries in the former Soviet Union. $J$ Public Health. 2013 Dec 1;35(4):548-557.

34 Carroll D, Phillips AC, Gale CR, Batty GD. Generalized anxiety and major depressive disorders, their comorbidity and hypertension in middle-aged men. Psychosom Med. 2010 Jan;72(1):16-19.

35 Christofaro DGD, Ritti-Dias RM, Chiolero A, Fernandes RA, Casonatto J, de Oliveira AR. Physical activity is inversely associated with high blood pressure independently of overweight in Brazilian adolescents. Scand J Med Sci Sports. 2013 Jun;23(3):317-322.

36 He FJ, Nowson CA, Lucas M, MacGregor GA. Increased consumption of fruit and vegetables is related to a reduced risk of coronary heart disease: meta-analysis of cohort studies. J Hum Hypertens. 2007 Sep;21(9):717-728.

37 Raja BK, Devi VNK. Prevalence of tobacco use among school-going adolescents in India: a systematic review of the literature. Cancer Res Stat Treat. 2018 Jul 1;1(2): 110.

38 GLOBAL ADULT TOBACCO SURVEY INDIA 2016-2017 Report Second Round [Internet]. New Delhi: Ministry of Health and Family Welfare, Government of India; 2018 Jun [cited 2021 Mar 19] Available from: https://ntcp.nhp.gov.in/assets/doc ument/surveys-reports-publications/Global-Adult-Tobacco-Survey-Second-RoundIndia-2016-2017.pdf.

39 Best Buys" And Other Recommended Interventions For The Prevention And Control Of Noncommunicable Diseases Updated ". APPENDIX 3 OF THE GLOBAL ACTION PLAN FOR THE PREVENTION AND CONTROL OF NONCOMMUNICABLE DISEASES 2013-2020; 2017 [Internet]. Word Health Organization; [cited 2021 Mar 19] Available from: https://www.who.int/ncds/management/WHO_Appendix_BestBuys. pdf. 